\title{
Adjuvant Chemotherapy for Patients with Breast Cancer Based on Clinical and Evolving Oncotype DX Criteria
}

\author{
Jamal Zekria ${ }^{a}$ Meteb Al-Foheidi ${ }^{b}$ Maaz Alatac ${ }^{c}$ Reem Zabani ${ }^{d}$ \\ Ayman Rasmy ${ }^{\mathrm{e}} \mathrm{f}$ \\ ${ }^{a}$ College of Medicine, Al-Faisal University, King Faisal Specialist Hospital \& Research Centre, Jeddah, Saudi Arabia; \\ bPrincess Noorah Oncology Center, King Saud bin Abdulaziz University, Jeddah, Saudi Arabia; ${ }^{\circ}$ King Faisal \\ Specialist Hospital \& Research Centre, Jeddah, Saudi Arabia; ${ }^{\mathrm{d}}$ Ibn Sina National Medical College, Jeddah, Saudi \\ Arabia; ${ }^{\mathrm{e}}$ Medical Oncology Department, King Saud Medical City, Riyadh, Saudi Arabia; ${ }^{\mathrm{f}}$ Medical Oncology, Zagazig \\ University Hospitals, Zagazig, Egypt
}

\section{Keywords}

Adjuvant treatment - Breast cancer · Oncotype DX

\begin{abstract}
Introduction: Oncotype DX assay recurrence score (ODX-RS) cut-off values have recently changed after the publication of the TAILOR-X trial results. We aim to explore decisions for adjuvant chemotherapy (ACT) based on physicians' clinical assessment and the evolving ODX-RS. Methodology: Patients who underwent ODX testing after curative surgical resection of estrogen receptor positive (ER+), Her2 non-overexpressed (Her2-) and lymph node-negative ( $\mathrm{LN}-$ ) breast cancer (BC) were eligible. Management of these patients was guided by the results of the old ODX-RS- $1(<18,18-30$, and $\geq 31$ ) risk grouping. For the purpose of this study, treatment decisions were also assumed according to TAILOR-X results (ODX-RS-2). Decisions of 3 medical oncologists on ACT were solicited by blinding them to the RS to investigate concordance with ODXA RS-1 and 2 recommendations. Results: Sixty-six consecutive patients were included. Median age was 50.5 (range: $21-73$ ) years. There was 1 male patient, and $37 / 65$ females (56.9\%) were premenopausal. Among the 3 oncologists, recommendations for ACT based on clinical assessment were discrepant in 29 (43.9\%) patients. Based on majority consensus ( $\geq 2$ oncologists), ACT would have been
\end{abstract}

recommended to $22 / 41(53.7 \%)$ and $22 / 46$ (47.82\%) patients with low-risk tumors according to ODX-RS-1 and ODX-RS-2, respectively. Compared to ODX-RS-1, ODX-RS-2 identifies $12 \%$ (46 vs. 41 ) more low-risk patients and $66 \%$ ( 20 vs. 12 patients) more high-risk patients. Conclusion: Overtreatment and discrepancies in the management of patients with ER+/ Her2-/LN- early BC can be minimized by the implementation of ODX genomic assay. Some differences in ACT recommendations exist between ODX-RS-1 and ODX-RS-2.

(c) 2020 S. Karger AG, Basel

\section{Introduction}

Globally, breast and lung cancers are the most commonly diagnosed malignancies, each representing about $11.6 \%$ of the total registered cases. In females, breast cancer (BC) accounts for $24.2 \%$ of all newly diagnosed female cancers [1].

About half to three-quarters of $\mathrm{BC}$ patients are diagnosed with localized stage I/II disease where regional lymph nodes (LNs) are not involved with malignant cells $[2,3]$. The vast majority of these patients will undergo curative intent surgical resection. Adjuvant hormonal therapy is recommended in about $75 \%$ of these patients as their tumors express estrogen receptor $(\mathrm{ER}+)[3,4]$. 
*Medical oncology clinic visit and treatment recommendation guided by ODX-RS-1

${ }^{*}$ Treatment in routine clinical setting

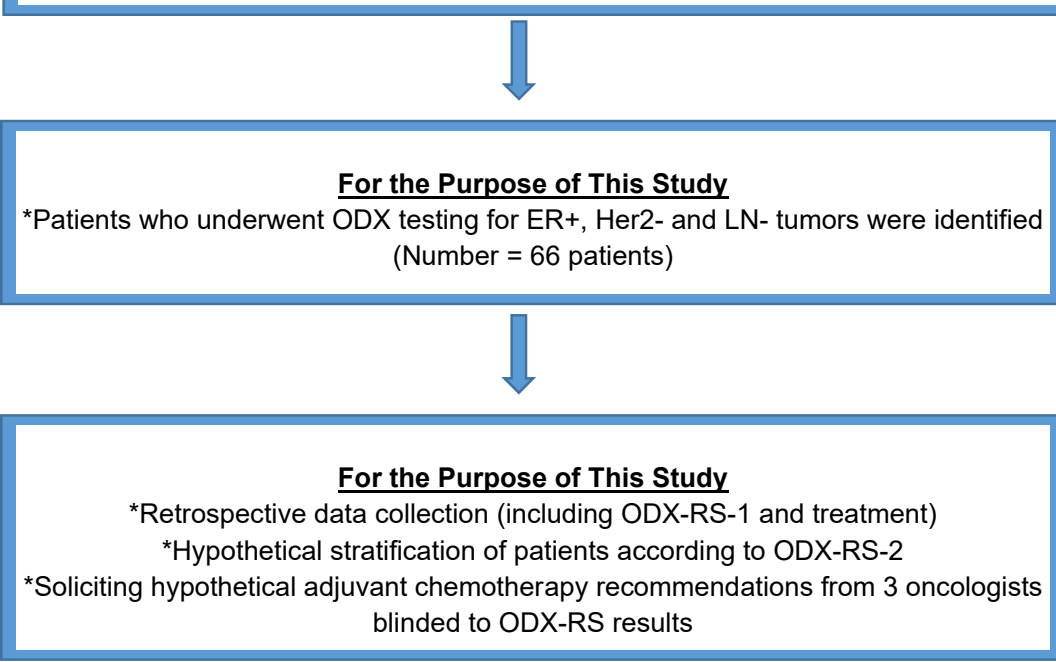

Fig. 1. Study scheme and management of patients in routine clinical setting.

Until recently, decisions for adjuvant chemotherapy (ACT) were solely based on clinical features such as patient's age, tumor size and grade, and LN involvement. These features and their thresholds for recommending ACT remained arbitrary and open to interpretation.

The oncotype DX (ODX) assay is a reverse-transcriptase polymerase chain reaction test based on the expression of 21 genes. The assay reports a recurrence score (RS) that is used to assign patients to groups with low risk (RS $<18$ ), intermediate risk (RS 18-30), and high risk (RS 231) of distant recurrence in patients with ER+, Her2 non-overexpressed (Her2-), and LN negative (LN-) BC [5].

The RS is also used to determine the likelihood of benefit from chemotherapy ACT. These cut-off values were validated from data of previously published trials.

More recently, the value of ODX assay was investigated prospectively in the TAILOR-X trial. Patients with midrange RS (11-25) were randomly assigned to receive either chemo-endocrine therapy or endocrine therapy alone. Results showed that ACT did not improve the outcome of these patients [6].

These results prompted the oncology community including the National Comprehensive Cancer Network (NCCN) to adopt a new algorithm based on modified ODX-RS cut-off thresholds dividing patients into 2 groups. Low-risk group with an RS of up to 15 (any age) and 16-25 (age $>50$ years) where ACT is not recommended. High-risk group was defined as RS $>25$ and 16-25 (age $\leq 50$ years) where ACT should be considered [7].

We aim to study the impact of adopting the ODX assay on treatment decisions in routine clinical practice in the context of the evolving RS thresholds.

\section{Patients and Methods}

All consecutive patients who underwent ODX assay testing after curative surgical resection including axillary LN procedure (sentinel biopsy, sampling or clearance) for ER+, Her2-, and LN$\mathrm{BC}$ were included. Patients with micrometastases to LNs and those who received preoperative systemic anti-cancer therapy were excluded. Decisions on ACT were guided in the routine clinical setting by the results of the ODX-RS $(<18,18-30$, and $\geq 31)$ risk grouping labelled as ODX-RS-1 (Table 1). All patients received adjuvant hormonal therapy. Data was collected retrospectively from the electronic and paper records from oncology units in 3 different hospitals. The first test was performed in July 2013 and the last in December 2017.

For the purpose of this study, treatment decisions were also assumed according to the new RS threshold (ODX-RS-2) guided by the results of TAILOR-X trial (Table 1). Decisions of 3 medical oncologists on ACT recommendations were solicited by blinding them to the RS values to investigate concordance with ODXA RS- 1 and 2 results. Majority opinion ( $\geq 2$ oncologists) was considered to represent their consensus recommendation. Figure 1 illustrates the management of patients in routine clinical setting and study methods. 
Table 1. Oncotype-DX threshold values

\begin{tabular}{lll}
\hline Risk groups & ODX-RS-1 [5] & ODX-RS-2 [6] \\
\hline Low & $<18$ & $\begin{array}{l}\leq 15 \text { (any age) and } 16-25 \\
\text { (age }>50 \text { years) }\end{array}$ \\
Intermediate & $18-30$ & $\begin{array}{l}\text { not applicable } \\
>25 \text { and } 16-25 \text { (age } \leq 50 \text { years) }\end{array}$ \\
High & $\geq 31$ & $>25$ \\
\hline
\end{tabular}

\section{Results}

Sixty-six consecutive patients fulfilled the inclusion criteria and none were excluded. Patients' characteristics are summarized in Table 2 . Twelve (18.8\%) patients received ACT guided by results of the established ODXRS-1 in the routine clinical setting (Table 3). Based on clinical assessment, the oncologists' consensus recommended ACT for 37 (56.06\%) of all patients. None of the 41 patients with low-risk ODX-RS-1 received ACT, while the oncologists' consensus recommended it for $53.65 \%$ of this subgroup. Similar results were observed when ODXRS-2 threshold was considered (Table 3).

In total, $12(18.8 \%)$ patients received ACT according to ODX-RS-1, while 20 patients would have received ACT according ODX-RS-2 (Table 3 ).

Based on clinical assessment, there was unanimous agreement among the 3 oncologist recommendations whether for or against ACT in 37 (56.1\%) patients with discrepant recommendations in the rest (43.9\%).

\section{Discussion}

For many decades, clinical decision-making about ACT for patients with early BC has been based on patientand tumor-related prognostic factors including patient's age, tumor size, grade and receptors, and LN status [8].

$\mathrm{ER}+$, Her2-, and LN- tumors are generally accepted to be associated with relatively favorable outcome. The benefit of ACT is modest at best, particularly in patients with these tumors. In practice, the majority of these patients will be overtreated and exposed to physical toxicity and financial burden of ACT with no improvement in BC-specific outcome [9-11].

A number of prognostic (and probably predictive) genomic assays have been developed over the last decade. The commercially available assays include ODX, Prosigna, Mammaprint, Breast Cancer Index, Genomic Grade Index, and EndoPredict. These tests have gained popularity and became widely adopted in clinical practice. This has changed the landscape of postoperative management of patients with $\mathrm{BC}$ over the recent few years [12].

ODX is a real-time, reverse transcriptase polymerase chain reaction assay that quantifies 16 cancer-related
Table 2. Patients' characteristics

\begin{tabular}{ll} 
Gender & \\
$\quad$ Female & $65(98.5)$ \\
$\quad$ Male & $1(1.5)$ \\
Age, years & $50.5(21-73)$ \\
Menopausal state & \\
$\quad$ Premenopausal & $37 / 65(56.9)$ \\
$\quad$ Postmenopausal & $28 / 65(43.1)$ \\
Histology & \\
$\quad$ Invasive ductal carcinoma & $57(86.4)$ \\
$\quad$ Invasive lobular carcinoma & $8(12.1)$ \\
$\quad$ Invasive papillary carcinoma & $1(1.5)$ \\
Tumor size, mm & $21.5(10-55)$ \\
T1 ( $\leq 20$ mm) & $32(48.5)$ \\
T2 $(20-50$ mm) & $33(50)$ \\
T3 (>50 mm) & $1(1.5)$ \\
Tumor grade & \\
I & $6(9.1)$ \\
II & $46(69.7)$ \\
III & $41(21.2)$ \\
Ki-67 & \\
$\quad$ Not reported & 41 \\
Reported & 25 \\
$\quad<14$ & $8 / 25(32)$ \\
$\geq 14$ & $17 / 25(68)$ \\
\hline
\end{tabular}

Data are presented as $n(\%)$ or median (range).

Table 3. Results showing adjuvant chemotherapy treatment and recommendations

\begin{tabular}{lccl}
\hline & $n$ & $\begin{array}{l}\text { Chemotherapy } \\
\text { treatment/ } \\
\text { recommendation }\end{array}$ & $\begin{array}{l}\text { Hypothetical chemo- } \\
\text { therapy recommen- } \\
\text { dation based on clini- } \\
\text { cal assessment }\end{array}$ \\
\hline ODX-RS-1 & & & \\
Low & 41 & $0 / 41(0 \%)$ & $22 / 41(53.65 \%)$ \\
Intermediate & 19 & $6 / 19(31.6 \%)$ & $11 / 19(57.89 \%)$ \\
High & 6 & $6 / 6(100 \%)$ & $4 / 6(66.66 \%)$ \\
Total & 66 & $12 / 66(18.18 \%)$ & $37 / 66(56.06 \%)$ \\
\hline ODX-RS-2 & & & $22 / 46(47.82 \%)$ \\
Low & 46 & $2 / 46(4.3 \%)^{*}$ & $15 / 20(75 \%)$ \\
High & 20 & $10 / 20(50 \%)^{*}$ & $37 / 66(56.06 \%)$ \\
Total & 66 & & \\
\hline
\end{tabular}

* Patients received ACT in real-life practice based on ODXRS-1.

genes and 5 reference genes in formalin-fixed paraffinembedded material to provide prognostic information. ODX is probably the most commonly used test to guide adjuvant treatment decisions for patients with $\mathrm{BC}$.

Our early experience with the implementation of ODX showed that $62 \%(41 / 66)$ of patients with ER+/Her2-/ $\mathrm{LN}$ - early BC are at low risk of distant recurrence (ODXRS-1 <18). Most previous studies demonstrated that 
more than half of patients have low RS. A pooled analysis of 565 patients from 4 European countries found that $55.2 \%$ have low RS [13].

Similar rates were reported in studies from different parts of the world such as Mexico (45\%), Hong Kong (50.7\%), United Arab Emirates (53.2\%), Turkey (57\%), and the United States (59\%) [14-18].

Prior to the era of implementing genomic assays, some practice guidelines recommended considering ACT for patients with tumor size $>5 \mathrm{~mm}$, in particular if there are other high-risk features such as intermediate or high histologic grade $[19,20]$.

The size of all tumors (including those with low RS) in our study was $>5 \mathrm{~mm}$ (Table 2). Consequently, ACT would have been considered for and administered to the majority of our patients if these practice guidelines were followed. Indeed, the majority opinion of the 3 oncologists recommended ACT for $53.65 \%$ of patients with low RS while none of them received ACT when ODX-RS-1 was considered.

The European Society of Medical Oncology (ESMO) 2008 guidelines define $\mathrm{LN}$ - tumors to be low risk if they have all the following features: $\leq 2 \mathrm{~cm}$, Grade I, absence of peritumoral vascular invasion, HER2 - and patient age $\geq 35$ years [21].

The 41 patients with low ODX-RS- 1 included 21 (51.2\%) with tumors $>2 \mathrm{~cm}$ in size and 37 (90.2\%) with grade II/III. The majority of these patients would have been considered for ACT based on ESMO 2008 guidelines.

Our findings confirm that the implementation of ODX assay can prevent the unnecessary administration of ACT in at least half of the patients (Table 3). Other studies have reported similar trends to de-escalation of treatment. In a study from Australia that included 235 patients with LN- BC, ACT was recommended to 89 patients, but only to 37 of them when the results of ODX assay were considered indicating treatment de-escalation in $58 \%$ of patients [22]. A study from the UK reported that $43 \%$ patients were spared chemotherapy when the results of the ODX assay were considered [23].

There is some disagreement on which traditional clinical factors are of prognostic and predictive importance. Certainly, defining the thresholds of these factors remains arbitrary and ambiguous.

At the St. Gallen Consensus Conference 2011, various degrees of disagreement existed when an expert international faculty discussed these factors. There were diverse votes for Ki-67, weak steroid hormone receptor expression, and presence of lymphovascular invasion as factors that influence the decision for chemotherapy [24]. This diversity continued to exist in the more recent $2017 \mathrm{St}$. Gallen consensus [25].

An example of a highly controversial factor is the Ki67. High Ki-67 index seem to be associated with worse outcome. However, there remain concerns about technical assessment and interobserver variability, uncertainties about the optimum cut-off value, and whether it can truly predict benefit from ACT [26-30].

Results of the Ki-67 index were available for only 25 patients in our study including 15 with low ODX-RS-1. Six out of 15 (40\%) had Ki-67 $\geq 14$ suggesting a luminal B-like genotype according to the St. Gallen guidelines, and thus it would have been acceptable to consider ACT for these patients [31]. Yet, the ODX assay defined these patients to have a low risk of recurrence, and they did not receive ACT. The most recent summary of the St. Gallen consensus published in 2019 discussed ACT without reference to Ki-67, probably in recognition of the role of the maturing data of multigene signature [32].

The ability of the ODX-RS to predict benefit from ACT was investigated in archival tumor tissues of patients treated in previous adjuvant trials. The results indicated that patients with low RS $(<18)$ tumors derived minimal, if any, benefit, while those with high RS $(\geq 31)$ gained larger benefit from ACT $[33,34]$.

The TAILOR-X trial enrolled women with hormone receptor positive, Her2- and LN- BC. Patients had to meet the NCCN guidelines for the recommendation of ACT, including a primary tumor size of $1.1-5.0 \mathrm{~cm}$ for a tumor of any grade or a size of $0.6-1.0 \mathrm{~cm}$ for a tumor of intermediate or high histologic grade or nuclear grade (or both). Of the 10,253 eligible women enrolled, 1,626 women had an RS of 0-10 and were assigned to receive endocrine therapy alone without ACT. In this patient population, the 5-year rate of invasive disease-free survival was $93.8 \%$, the rate of freedom from recurrence of distant recurrence was 99.3, and the rate of overall survival was 98.0\%. The investigators concluded that these patients may be safely spared ACT [35].

Patients with midrange RS (11-25) were randomly assigned to receive either chemo-endocrine therapy or endocrine therapy alone. In these 6,711 patients, the addition of ACT did not significantly improve the outcome. Some benefit was observed in women 50 years of age or younger with an RS of 16-25 [6].

The results of TAILOR-X set new RS cut-off values for the recommendation of ACT (Table 2).

In our study, 12 (18.18\%) received ACT based on ODXRS-1. These included 6/19 (31.6\%) patients with intermediate-risk RS-1 who were deemed to have unfavorable clinical features, and all $6(100 \%)$ patients with high-risk RS-1. On one hand, ODX-RS-2 identifies 5 more patients with low risk ( 46 vs. 41 ), of whom 2 patients received ACT that can be argued as unnecessary if ODX-RS-2 is implemented. On the other hand, ODX-RS-2 identifies 14 more patients with high risk (20 vs. 6) some of whom can be argued to be over treated if ODX-RS-2 is implemented (Table 3). In general, such arguments are expected as on- 
cologists start implementing the new ODX-RS-2. It is immensely reassuring that the ODX-RS- 2 is based on the results of the TAILOR-X trial which is a well-designed large prospective randomized clinical trial.

ODX-RS- 2 has the advantage of eliminating the intermediate-risk group and thus provides a much more practical guidance to physicians and patients in routine clinical settings. There remains ambiguity on whether ACT provides benefit for patients with an RS of 26-30. In this respect, the NCCN guidelines state that the omission of ACT has not been studied prospectively for these patients and that physicians should consider clinical and pathological factors with regard to the addition of ACT [7].

In principle, it is acceptable that the recommendation of ACT for women with ER+/Her2-/LN- early BC can be linked to increasing tumor size, higher grade, and younger patient's age. However, these are continuous factors representing a scale of risk. For each of these factors, identifying a dichotomizing point above which the risk is high and below which the risk is low has proven to be a challenge. For example, tumor size has been dichotomized at $0.5,1$, and $2 \mathrm{~cm}$ by different authorities and experts. As a consequence, it is expected that a patient may get different ACT recommendations by different physicians. A study investigating the frequency of ACT use for hormone receptor-positive, LN- BC from 1997 to 2004 at eight NCCN institutions showed that there did not seem to be consensus regarding which women should get ACT [36].

Our results confirm the lack of consensus as $43.9 \%$ of our patients' scenarios received different hypothetical recommendations by the 3 oncologists when guided only by the clinical and pathological factors (without ODX-RS). The implementation of $\mathrm{BC}$ genomic assays (including ODX) is likely to minimize discrepancies and increase oncologists' and patients' decision-making confidence [37].

\section{Conclusion}

Decisions on ACT for patients with ER+/Her2-/LNearly BC should be guided by ODX-RS to prevent overtreatment and discrepancies in management. Broadly, some differences in ACT recommendations exist between ODX-RS-1 and ODX-RS-2. These differences may carry some cost implications that need to be studied in larger cohorts.

\section{Acknowledgement}

The authors acknowledge the role of the 3 medical oncologists (Dr. Hossam Abdelrahman, Dr. Haleem Rasool, and Dr. Adnan Hussain) who provided hypothetical recommendations on ACT based on clinical factors. The authors also acknowledge the contribution of Dr. Yasser Sorour and Dr. Miral Mashhour in collecting some of the patient clinical data.

\section{Statement of Ethics}

Relevant human research regulatory bodies including the institutional review board have granted approval to conduct this research protocol. Patient consent was not necessary due to the retrospective and noninterventional design of the study. This research did not involve any animal experiments.

\section{Disclosure Statement}

All authors declare no conflict of interest.

\section{Funding Sources}

This research received no specific grant from any funding agency in the public, commercial, or not-for-profit sectors.

\section{Author Contributions}

Jamal Zekri: major role in the conception and design of the study, data collection, data analysis and data interpretation. Seeking regulatory approval. Contribution to the progress and revision of the work. Major contribution to the manuscript writing. Approval of the final manuscript version. Meteb Al-Foheidi: contribution to the design of the study, data collection, and data interpretation. Seeking regulatory approval. Contribution to the progress and revision of the work. Critically revising the manuscript writing at different steps. Final approval of the version. Maaz Alata: Contribution to the design of the study, data collection, data analysis and data interpretation. Contribution to the manuscript writing. Approval of the final manuscript version. Reem Zabani: Acquisition, analysis and interpretation of the data. Contribution to the manuscript writing. Approval of the final manuscript version. Ayman Rasmy: Major role in the conception and design of the study, data collection, data analysis and data interpretation. Seeking regulatory approval. Contribution to the progress and revision of the work. Critically revising the manuscript writing at different steps. Final approval of the version.

\section{References}

1 Bray F, Ferlay J, Soerjomataram I, Siegel RL, Torre LA, Jemal A. Global cancer statistics 2018: GLOBOCAN estimates of incidence and mortality worldwide for 36 cancers in 185 countries. CA Cancer J Clin. 2018 Nov;68(6): 394-424.

2 Celaya MO, Berke EM, Onega TL, Gui J, Riddle BL, Cherala SS, et al. Breast cancer stage at diagnosis and geographic access to mammography screening (New Hampshire, 19982004). Rural Remote Health. 2010 Apr-Jun; 10(2):1361. [cited 2019 Jun 3].

3 Holleczek B, Jansen L, Brenner H. Breast cancer survival in Germany: a population-based high resolution study from Saarland. PLoS One. 2013 Jul;8(7):e70680. 
4 Dunnwald LK, Rossing MA, Li CI. Hormone receptor status, tumor characteristics, and prognosis: a prospective cohort of breast cancer patients. Breast Cancer Res. 2007;9(1):R6.

5 Paik S, Shak S, Tang G, Kim C, Baker J, Cro$\operatorname{nin} \mathrm{M}$, et al. A multigene assay to predict recurrence of tamoxifen-treated, node-negative breast cancer. N Engl J Med. 2004 Dec; 351(27):2817-26

6 Sparano JA, Gray RJ, Makower DF, Pritchard KI, Albain KS, Hayes DF, et al. Adjuvant Chemotherapy Guided by a 21-Gene Expression Assay in Breast Cancer. N Engl J Med. 2018 Jul;379(2):111-21.

7 Gradishar WJ, Abraham J, Aft R, Agnese D, Allison KH, Blair SL, et al. NCCN Guidelines Version 1.2019 Breast Cancer NCCN Evidence Blocks. 2019 [cited 2019 Jun 3]. Available from: https://www.nccn.org/professionals/physician_gls/pdf/breast_blocks.pdf

8 Stiggelbout AM, de Haes JC, van de Velde CJ. Adjuvant chemotherapy in node negative breast cancer: patterns of use and oncologists' preferences. Ann Oncol. 2000 May;11(5): 631-3.

9 Gelber RD, Cole BF, Goldhirsch A, Rose C, Fisher B, Osborne CK, et al. Adjuvant chemotherapy plus tamoxifen compared with tamoxifen alone for postmenopausal breast cancer: meta-analysis of quality-adjusted survival. Lancet. 1996 Apr;347(9008):1066-71. [cited 2019 Jun 3].

10 Early Breast Cancer Trialists' Collaborative Group (EBCTCG). Effects of chemotherapy and hormonal therapy for early breast cancer on recurrence and 15-year survival: an overview of the randomised trials. Lancet. 2005 May;365(9472):1687-717.

11 Peto R, Davies C, Godwin J, Gray R, Pan HC, Clarke M, et al.; Early Breast Cancer Trialists' Collaborative Group (EBCTCG). Comparisons between different polychemotherapy regimens for early breast cancer: meta-analyses of long-term outcome among 100,000 women in 123 randomised trials. Lancet. 2012 Feb;379(9814):432-44.

12 Nguyen MT, Stessin A, Nagar H, D'Alfonso TM, Chen Z, Cigler T, et al. Impact of oncotype DX recurrence score in the management of breast cancer cases. Clin Breast Cancer. 2014 Jun; 14(3):182-90.

13 Albanell J, Svedman C, Gligorov J, Holt SD, Bertelli G, Blohmer JU, et al. Pooled analysis of prospective European studies assessing the impact of using the 21-gene Recurrence Score assay on clinical decision making in women with oestrogen receptor-positive, human epidermal growth factor receptor 2-negative early-stage breast cancer. Eur J Cancer. 2016 Oct; 66:104-13.

14 Bargallo JE, Lara F, Shaw-Dulin R, Perez-Sánchez V, Villarreal-Garza C, Maldonado-Martinez $\mathrm{H}$, et al. A study of the impact of the 21gene breast cancer assay on the use of adjuvant chemotherapy in women with breast cancer in a Mexican public hospital. J Surg Oncol. 2015 Feb;111(2):203-7.
15 Leung RC, Yau TC, Chan MC, Chan SW, Chan TW, Tsang YY, et al. The Impact of the Oncotype DX Breast Cancer Assay on Treatment Decisions for Women With Estrogen Receptor-Positive, Node-Negative Breast Carcinoma in Hong Kong. Clin Breast Cancer. 2016 Oct;16(5):372-8.

16 Jaafar H, Bashir MA, Taher A, Qawasmeh K, Jaloudi M. Impact of Oncotype DX testing on adjuvant treatment decisions in patients with early breast cancer: a single-center study in the United Arab Emirates. Asia Pac J Clin Oncol. 2014 Dec;10(4):354-60.

17 Ozmen V, Atasoy A, Gokmen E, Ozdogan M, Guler N, Uras C, et al. Impact of Oncotype DX Recurrence Score on Treatment Decisions: Results of a Prospective Multicenter Study in Turkey. Cureus. 2016 Mar;8(3):e522.

18 Hanna MG, Bleiweiss IJ, Nayak A, Jaffer S. Correlation of Oncotype DX Recurrence Score with Histomorphology and Immunohistochemistry in over 500 Patients. Int J Breast Cancer. 2017;2017:1257078.

19 Carlson RW, Brown E, Burstein HJ, Gradishar WJ, Hudis CA, Loprinzi C, et al.; National Comprehensive Cancer Network. NCCN Task Force Report: Adjuvant Therapy for Breast Cancer. J Natl Compr Canc Netw. 2006 Mar; 4 Suppl 1:S1-26. [cited 2019 Jun 3].

20 Eisen A, Fletcher GG, Gandhi S, Mates M, Freedman OC, Dent SF, et al; Members of the Early Breast Cancer Systemic Therapy Consensus Panel. Optimal systemic therapy for early breast cancer in women: a clinical practice guideline. Curr Oncol. 2015 Mar;22(Suppl 1):S67-S81.

21 Pestalozzi B, Castiglione M; ESMO Guidelines Working Group. Primary breast cancer: ESMO clinical recommendations for diagnosis, treatment and follow-up. Ann Oncol. 2008 May;19(Suppl 2):ii7-10.

22 Chin-Lenn L, De Boer RH, Segelov E, Marx GM, Hughes TM, McCarthy NJ, et al. The impact and indications for Oncotype DX on adjuvant treatment recommendations when third-party funding is unavailable. Asia Pac J Clin Oncol. 2018 Dec;14(6):410-6.

23 Khan MA, Henderson L, Clarke D, Harries S, Jones L. The Warwick Experience of the Oncotype DX ${ }^{\circledR}$ Breast Recurrence Score ${ }^{\circledR}$ Assay as a Predictor of Chemotherapy Administration. Breast Care (Basel). 2018 Oct;13(5):369-72.

24 Gnant M, Harbeck N, Thomssen C. St. Gallen 2011: Summary of the Consensus Discussion. Breast Care (Basel). 2011;6(2):136-41.

25 Gnant M, Harbeck N, Thomssen C. St. Gallen/Vienna 2017: A Brief Summary of the Consensus Discussion about Escalation and De-Escalation of Primary Breast Cancer Treatment. Breast Care (Basel). 2017 May; 12(2):102-7.

26 Viale G, Giobbie-Hurder A, Regan MM, Coates AS, Mastropasqua MG, Dell'Orto P, et al.; Breast International Group Trial 1-98. Prognostic and predictive value of centrally reviewed Ki-67 labeling index in postmenopausal women with endocrine-responsive breast cancer: results from Breast International Group Trial 1-98 comparing adjuvant tamoxifen with letrozole. J Clin Oncol. 2008 Dec;26(34):5569-75.
27 Fasching PA, Gass P, Häberle L, Volz B, Hein A, Hack CC, et al. Prognostic effect of Ki-67 in common clinical subgroups of patients with HER2-negative, hormone receptor-positive early breast cancer. Breast Cancer Res Treat. 2019 Jun;175(3):617-25.

28 Colomer R, Aranda-López I, Albanell J, García-Caballero T, Ciruelos E, López-García MÁ, et al. Biomarkers in breast cancer: A consensus statement by the Spanish Society of Medical Oncology and the Spanish Society of Pathology. Clin Transl Oncol. 2018 Jul;20(7): 815-26.

29 Viale G, Regan MM, Mastropasqua MG, Maffini F, Maiorano E, Colleoni M, et al.; International Breast Cancer Study Group. Predictive value of tumor Ki-67 expression in two randomized trials of adjuvant chemoendocrine therapy for node-negative breast cancer. J Natl Cancer Inst. 2008 Feb;100(3):207-12.

30 Sutepvarnon A, Warnnissorn M, Srimuninnimit V. Predictive value of Ki67 for adjuvant chemotherapy in node-negative, hormone receptor-positive breast cancer. J Med Assoc Thai. 2013 Feb;96 Suppl 2:S60-6. [cited 2019 Jun 3].

31 Goldhirsch A, Winer EP, Coates AS, Gelber RD, Piccart-Gebhart M, Thürlimann B, et al.; Panel members. Personalizing the treatment of women with early breast cancer: highlights of the St Gallen International Expert Consensus on the Primary Therapy of Early Breast Cancer 2013. Ann Oncol. 2013 Sep;24(9): 2206-23.

32 Balic M, Thomssen C, Würstlein R, Gnant M, Harbeck N. St. Gallen/Vienna 2019: A brief summary of the consensus discussion on the optimal primary breast cancer treatment. Breast Care (Basel). 2019 Apr;14(2):103-10.

33 Paik S, Tang G, Shak S, Kim C, Baker J, Kim $\mathrm{W}$, et al. Gene expression and benefit of chemotherapy in women with node-negative, estrogen receptor-positive breast cancer. J Clin Oncol. 2006 Aug;24(23):3726-34.

34 Albain KS, Barlow WE, Shak S, Hortobagyi GN, Livingston RB, Yeh IT, et al.; Breast Cancer Intergroup of North America. Prognostic and predictive value of the 21-gene recurrence score assay in postmenopausal women with node-positive, oestrogen-receptor-positive breast cancer on chemotherapy: a retrospective analysis of a randomised trial. Lancet Oncol. 2010 Jan;11(1):55-65.

35 Sparano JA, Gray RJ, Makower DF, Pritchard KI, Albain KS, Hayes DF, et al. Prospective Validation of a 21-Gene Expression Assay in Breast Cancer. N Engl J Med. 2015 Nov; 373(21):2005-14

36 Hassett MJ, Hughes ME, Niland JC, Edge SB, Theriault RL, Wong YN, et al. Chemotherapy use for hormone receptor-positive, lymph node-negative breast cancer. J Clin Oncol. 2008 Dec;26(34):5553-60.

37 Fallowfield L, Matthews L, May S, Jenkins V, Bloomfield D. Enhancing decision-making about adjuvant chemotherapy in early breast cancer following EndoPredict testing. Psychooncology. 2018 Apr;27(4):1264-9.
Adjuvant Chemotherapy

Recommendations in Breast Cancer 